Ann. Sci. forest., 1973, 30 (2), 127-140.

\title{
INFLUENCE DE L'ÉLAGAGE DE BRANCHES VIVANTES SUR LA STRUCTURE DES ACCROISSEMENTS ANNUELS ET SUR QUELQUES CARACTÉRISTIQUES DU BOIS DE DOUGLAS ET DE GRANDIS
}

\author{
H. POLGE, R. KELLER et F. THIERCELIN \\ Station de Recherches sur la Qualité des bois, \\ Centre national de recherches forestieres, I. N. R. A., \\ Champenoux 54370 Einville
}

\begin{abstract}
RÉSUMÉ
Un élagage sévère a pour effet de réduire la progressivité du passage du bois initial au bois final et d'augmenter la densité moyenne, la densité maximale annuelle, la densité minimale annuelle, ainsi que le nombre et l'ampleur des doubles cernes.

La rétractibilité est accrue dans le sens tangentiel et dans le sens radial, mais légèrement diminuée dans le sens longitudinal.

D'une façon générale, pour un même taux d'élagage, les effets sont plus marqués sur le Grandis que sur le Douglas.
\end{abstract}

\section{1. - OBSERVATIONS PRÉLIMINAIRES}

De nombreuses publications ont déjà été consacrées aux divers effets de l'élagage :

Les unes concernent la conséquence la plus immédiate de cette opération, c'est-à-dire l'amélioration du classement des produits liée à la disparition des nœuds (NAGELI, 1952 Venet, 1955 et 1957 - Fielding, 1964 - Feinl et Godin, 1965 - Brown, 1965 - Callin, 1965 - KANO et al., 1969 - GAva, 1969).

D'autres ont trait à des effets moins connus et propres aux seuls élagages de branches vivantes : amélioration de la cylindricité (LEHTPERE, 1957 - LARSON, 1961-1962 et 1966 Polge, 1967 - Keller, 1968 - Polge, 1969), ou réduction de certains défauts caractéristiques du bois juvénile, comme sa faible densité, l'importance de la fibre torse et la déformation des débits qui en sont tirés (GerisCHER et DE VILLIERs, 1963). 
Dans le présent compte rendu, seront étudiés d'autres influences jusqu'ici méconnues de l'élagage, à savoir celles qui concernent la structure interne des accroissements annuels, telle que l'on peut l'analyser sur les courbes de variation de la densité du bois d'une part, et les différents retraits d'autre part, ainsi que la densité globale.

\section{2. - MATÉRIEL ET MÉTHODE}

\section{1. - Dispositif expérimental}

Les arbres étudiés font partie d'un dispositif expérimental d'élagage implanté au cours de la période d'arrêt de la végétation en 1966-1967 dans une plantation mélangée de Pseudotsuga menziesii et d'Abies grandis alors âgée de 13 ans et réalisée à l'écartement de $5 \mathrm{~m} \times 5 \mathrm{~m}$ (propriété de Maître Gallais à Saint-Christophe-de-Chaulieu, Calvados); ce dispositif comprend au total 80 arbres répartis à égalité entre les deux essences : douglas et grandis, et entre cinq traitements : témoins et arbres élagués à 20,30, 40 et $50 \%$. Du fait de la faible densité de plantation, tous les arbres étaient, lors de l'implantation du dispositif, porteurs de branches vertes sur toute la longueur du fût.

En fait, les déterminations décrites ci-dessous n'ont été faites que sur les traitements extrêmes, c'est-à-dire sur les 8 témoins et les 8 arbres élagués à $50 \%$ de chaque espèce.

Le matériel utilisé a consisté en carottes de sondage de $5 \mathrm{~mm}$ de diamètre prélevées à hauteur d'homme suivant un orientement constant, mais en évitant le cas échéant le bois de compression ou les zones de nœuds.

\section{2. - Étude de la structure interne des accroissements annuels}

Toutes les carottes de sondage ont été radiographiées à l'aide d'un tube à rayons $\mathrm{X}$ à fenêtre de béryllium en utilisant les caractéristiques d'exposition suivantes :

- distance source-film : $2,50 \mathrm{~m}$,

— intensité du flux électronique : $20 \mathrm{~mA}$,

- tension accélératrice : $15 \mathrm{kV}$,

- temps d'exposition : 4 minutes.

Les images obtenues ont été explorées au microdensitomètre à l'aide d'un coin radiographique G2; le faisceau analyseur avait une largeur de 100 microns dans le sens radial.

Sur les enregistrements faits avec un rapport d'agrandissement de $4 \mathrm{X}$, ont été relevées les densités maximales et minimales annuelles, ainsi que les largeurs de cernes, caractéristiques maintenant

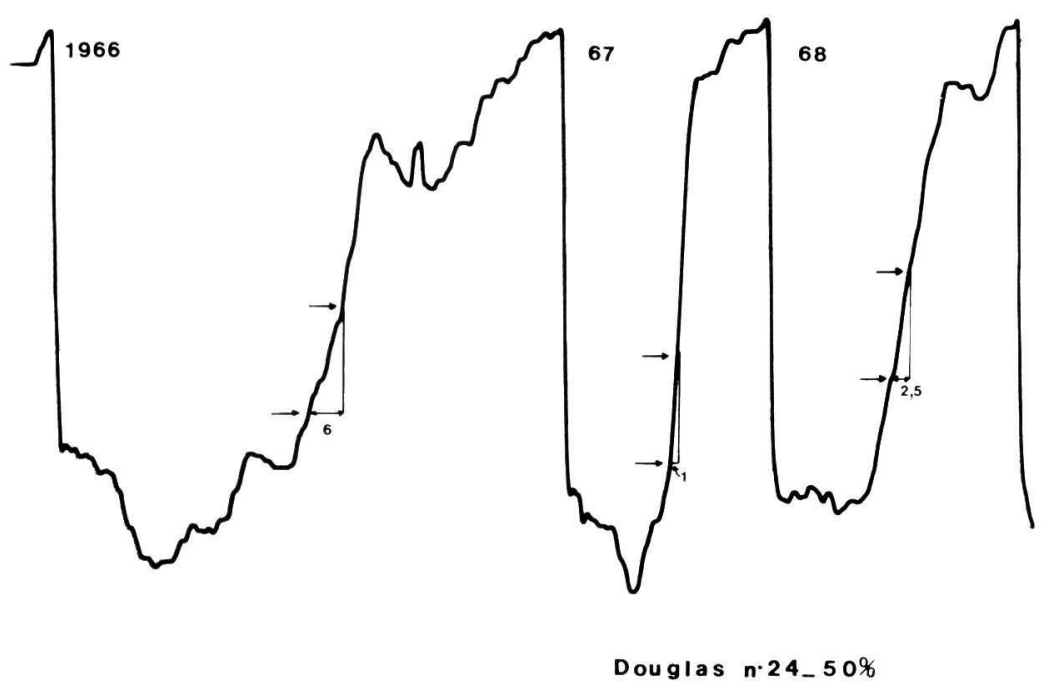

FIG. 1. - Exemples de l'indice de progressivité

FIG. 1. - Progressivity index examples 


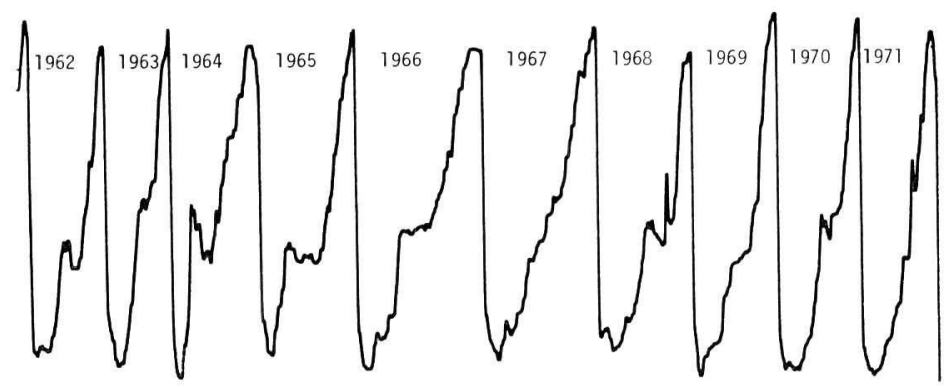

Douglas $n^{\circ} 21$ - témoin

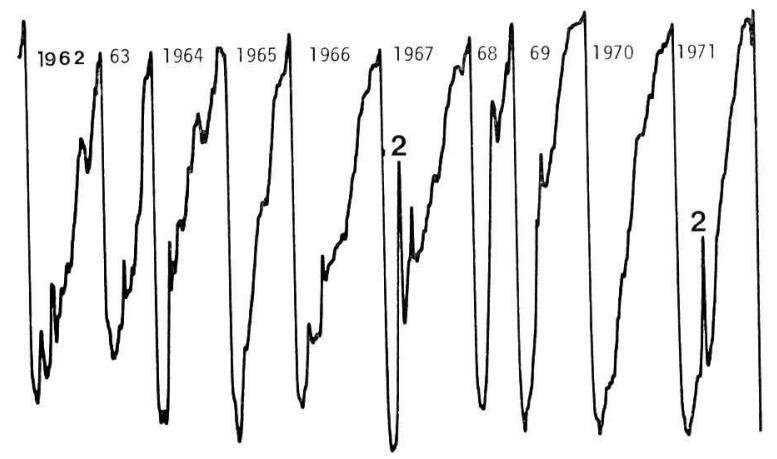

Douglas $n^{2} 30-50 \%$

FIG. 2. - Profils-types de douglas avec indication des notes de doubles cernes différentes de zéro FIG. 2. - Typical records of Douglas fir with false ring index (zero excluded)
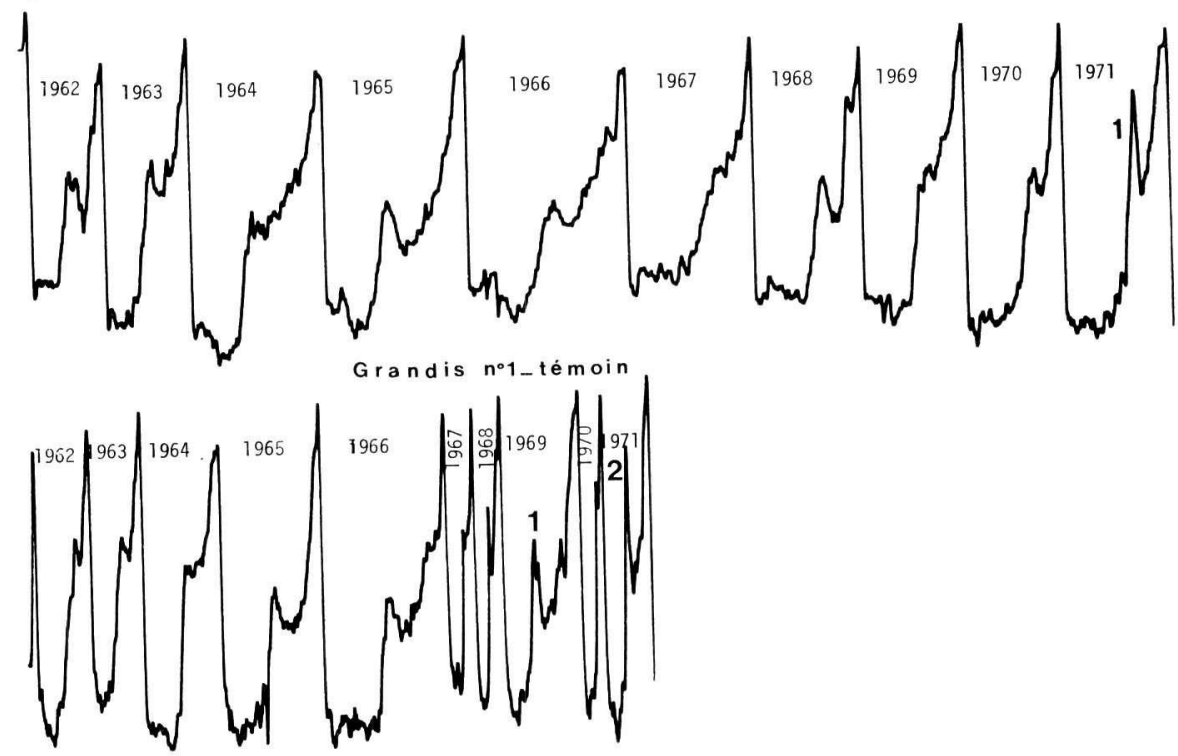

Grandis $n^{\circ} 12-50 \%$

FIG. 2bis. - Profils-types de grandis avec indication des notes de doubles cernes différentes de zéro FIG. 2bis. - Typical records of Grand fir with false ring index (zero excluded). 
classiques auxquelles ont été rajoutées deux autres non encore utilisées jusqu'ici : l'indice de progressivité du passage du bois initial au bois final et la note de fréquence - ampleur des doubles cernes.

L'indice de progressivité est l'inverse d'une pente mesurée sur l'enregistrement densitométrique; il est égal à la plus petite valeur de la différence d'abscisse se traduisant par une différence en densité du bois de $100 \mathrm{~g} / \mathrm{dm}^{3}$, la mesure étant faite dans la moitié inférieure du profil de chaque année (figure 1).

La note de doubles cernes a été calculée de la façon suivante : chaque accroissement annuel a été noté 0 lorsque le profil ne comportait aucune baisse de densité égale ou supérieure à $78 \mathrm{~g} / \mathrm{dm}^{3}$ (représentant à l'échelle $15 \mathrm{~mm}$ sur le graphique); la note 1 a été donnée aux baisses de densités comprises entre $78 \mathrm{~g} / \mathrm{dm}^{3}$ et $130 \mathrm{~g} / \mathrm{dm}^{3}(25 \mathrm{~mm}$ sur le graphique $)$ et la note 2 aux doubles cernes se traduisant par une baisse supérieure à $130 \mathrm{~g} / \mathrm{dm}^{3}$.

Sur les figures 2 et 2 bis ont été regroupés les profils-types d'un témoin et d'un arbre élagué pour chacune des deux espèces sur lesquels les notes chiffrées des doubles cernes sont indiquées en
gros caractères.

La note sur laquelle ont porté les calculs est une note moyenne annuelle calculée à partir des notes individuelles des 8 arbres de chaque essence et de chaque traitement. Toutes ces déterminations ont été faites sur 10 ans, à savoir les cinq dernières années avant celle de l'élagage (1962 à 1966) et
les cinq années suivantes.

\section{3. - Étude de la rétractibilité}

Les différents retraits ont été déterminés, sur les mêmes groupes d'accroissements annuels que ceux utilisés pour l'étude de la structure interne du bois, entre l'état saturé et l'état sec à l'air à $10 \%$ d'humidité.

Le présent compte-rendu étant le premier où il est fait état de telles mesures effectuées sur carottes de sondage, la méthodologie employée va être décrite ci-dessous avec quelques détails :

L'échantillon dont les dimensions axiales et tangentielles sont voisines de $5 \mathrm{~mm}$ repose sur une gouttière de $10 \mathrm{~mm}$ de large au milieu de laquelle se trouve le système de mesure (voir figure 3 ); celui-ci comporte une butée fixe, mais réglable, et une butée mobile solidaire d'un capteur de déplacement électronique; chaque butée est munie d'une touche-couteau cylindrique de $1 \mathrm{~mm}$ de diamètre, et la carotte est positionnée de façon telle que le fil du bois soit parallèle aux touches pour les mesures de retrait tangentiel, et perpendiculaire pour les mesures de retrait axial. Un certain jeu a été laissé volontairement dans l'ensemble du système de guidage pour que, dans le cas où l'échantillon comporte quelques légères sinuosités, il puisse se déplacer sous la pression du capteur (qui comporte un ressort taré à $45 \mathrm{~g}$ ), de façon à se présenter entre touches sous sa plus petite dimension dans le plan consi-
déré.

L'échantillon est poussé dans sa gouttière à l'aide d'une vis sans fin que commande une manivelle par l'intermédiaire d'un ensemble pignon - roue dentée; un tour de manivelle correspond à un déplacement de $1,45 \mathrm{~mm}$ et l'on procède à une mesure tous les tours de manivelle, tous les $1 / 2$ ou tous les $1 / 4$ de tours, suivant la longueur de l'échantillon, de façon à obtenir au total une centaine de valeurs pour chaque état de siccité et pour chaque direction.

Compte tenu du fait que le retrait affecte différemment le bois initial et le bois final, ce grand nombre de points de mesure est nécessaire si l'on veut obtenir des valeurs moyennes statistiquement valables, sans qu'il soit nécessaire de faire en sorte que les dimensions axiales ou tangentielles soient prises aux mêmes emplacements à l'état saturé et à l'état sec à l'air.

Le capteur de déplacement comporte un transformateur différentiel dont la tension de sortie est rigoureusement proportionnelle à l'enfoncement de la touche à raison d'un millivolt par micron, soit encore 2 volts pour l'amplitude totale de variation qui est de $2 \mathrm{~mm}$.

Les dimensions mesurées sont affichées au micron près sur un millivoltmètre numérique, luimême couplé à un télétype qui perfore sur ruban les valeurs des points de mesure et en fournit de plus un listage complet. L'interface qui les relie l'un à l'autre a été conçu de façon à faire précéder chaque indication du millivoltmètre par le numéro d'ordre de la mesure; il est ainsi possible d'obtenir facilement sur ordinateur, non seulement les valeurs moyennes et écarts-types des dimensions à un état de siccité et dans une direction donnée, mais encore d'en suivre les variations le long de l'échantillon par des calculs de régression linéaire ou polynômiale, les indications nécessaires au traitement des données étant frappées sur le clavier du télétype, et par suite inscrites sur bande perforée.

En l'état actuel de la méthodologie, l'appareil de mesure fonctionne de façon semi-automatique, c'est-à-dire qu'un opérateur intervient pour faire avancer l'échantillon et pour mettre en route 1'ensemble affichage - impression et perforation en appuyant sur une touche qui ferme le circuit du millivoltmètre.

Des tests de répétibilité ont montré que la précision sur la moyenne des mesures pour un échantillon varie selon les individus, les espèces, les directions et les états de siccité entre 1 et 5 microns; le 
temps de mesure est très court puisqu'il est possible de déplacer l'échantilion pendant l'impression et la perforation des résultats au point précédent; il se situe aux environs de 3 minutes pour 100 mesures.

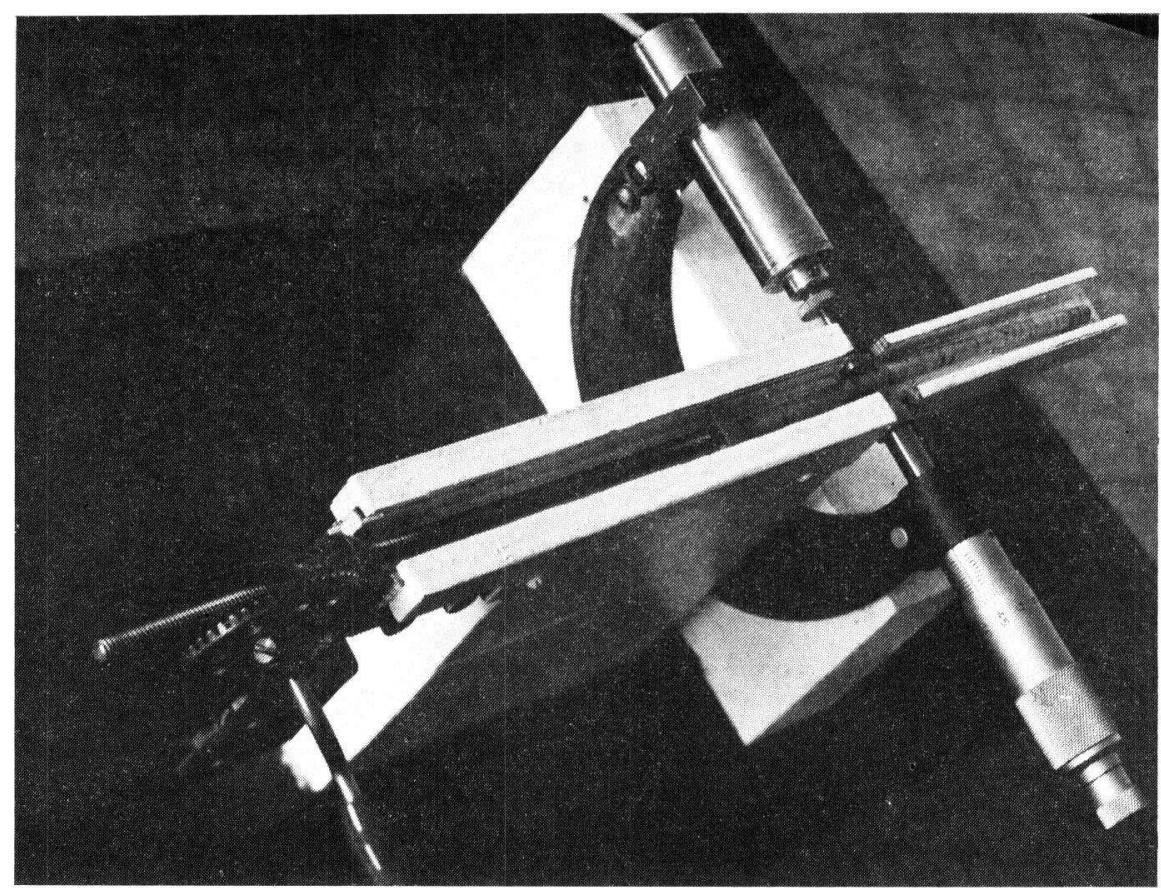

Fig. 3

La méthodologie utilisée pour les déterminations du retrait radial nécessite beaucoup moins de développement : les mesures sont faites au $1 / 100^{\circ}$ de $\mathrm{mm}$ près à l'aic'e, d'un micromètre de grande capacité; la précision est meilleure si l'on prend soin de découper les deux extrêmités de la carotte suivant des plans bien perpendiculaires à son axe; en outre, l'échantillon repose dans une gouttière en forme de $\mathrm{V}$, ce qui permet à l'opśrateur, par une simple fression des doigts de le rendre rectiligne au cas où il aurait tendance à se déformer.

\section{3. - RÉSULTATS}

\section{1. - Structure des accroissements annu?/s}

Les résultats concernant les diverses variables analysées à ce titre sont tous donnés sous forme de graphiques dans lesquels les variations relatives aux témoins sont représentées en traits pleins et celles relatives aux arbres élagués en traits interrompus; la date de l'élagage est rappelée sous forme d'un double trait vertical entre l'année 1966 et l'année 1967.

Des tests de comparaison de moyennes ont été faits pour toutes les années et toutes les caractéristiques; lorsqu'une différence est significative, elle est indiquée à l'aide d'un signe conventionnel entre les deux courbes représentatives :

- une double croix traduit une différence significative au seuil de $1 \%$,

- une croix simple, une différence significative au seuil de $5 \%$,

— un point, une différence significative au seuil de $10 \%$, cette dernière indication étant 
fournie contrairement aux usages pour indiquer une tendance dont on peut penser que l'absence de signification n'est due qu'au trop petit nombre d'échantillons (14 degrés de liberté).

\subsection{Largeur de cernes}

Pour les deux espèces (voir figure 4), un élagage sévère entraîne une diminution de la largeur des accroissements annuels qui est d'autant plus nette que les prélèvements étudiés ont été faits à hauteur d'homme, c'est-à-dire au niveau où la réduction de croissance est le plus sensible; on voit que la perte de vigueur est beaucoup plus sévère et beaucoup plus forte chez le grandis que chez le douglas où seule subsiste, dès la troisième année qui suit l'élagage, une tendance non significative à une largeur de cernes plus faible sur les arbres élagués que sur les témoins.
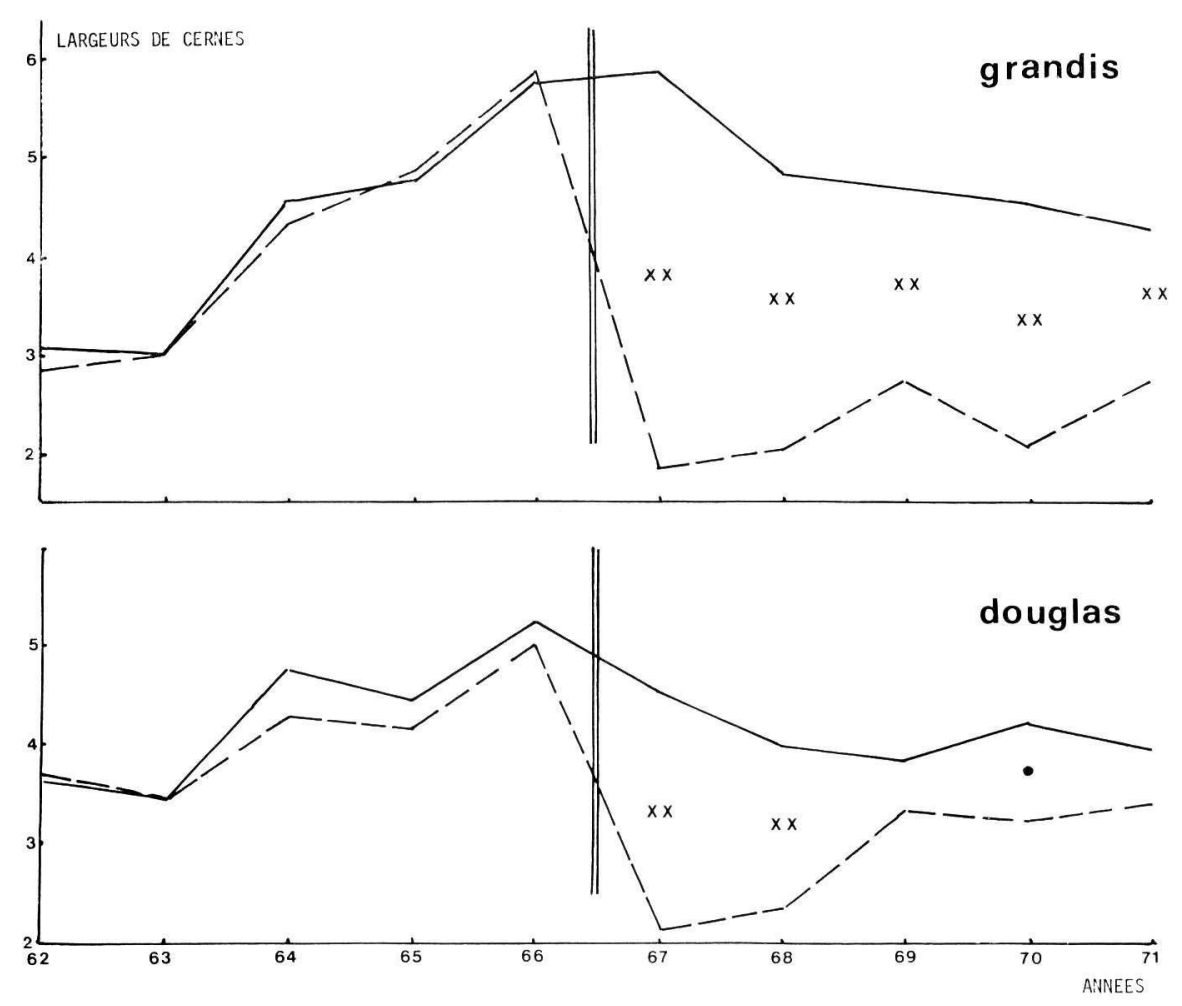

Fig. 4

\subsection{Densité maximale annuelle (figure 5)}

Les grandis élagués avaient malheureusement dès avant traitement une densité maximale supérieure aux témoins, avec une différence qui était significative en 1964; mais si l'on observe que l'écart était extrêmement réduit en 1966, c'est-à-dire au cours de la dernière année précédant l'opération, on voit que l'ablation de branches vivantes a entraîné une hausse des densités maximales annuelles, hausse qui s'est avérée très significative en 1968 et qui s'est encore maintenue jusqu'en 1971. 

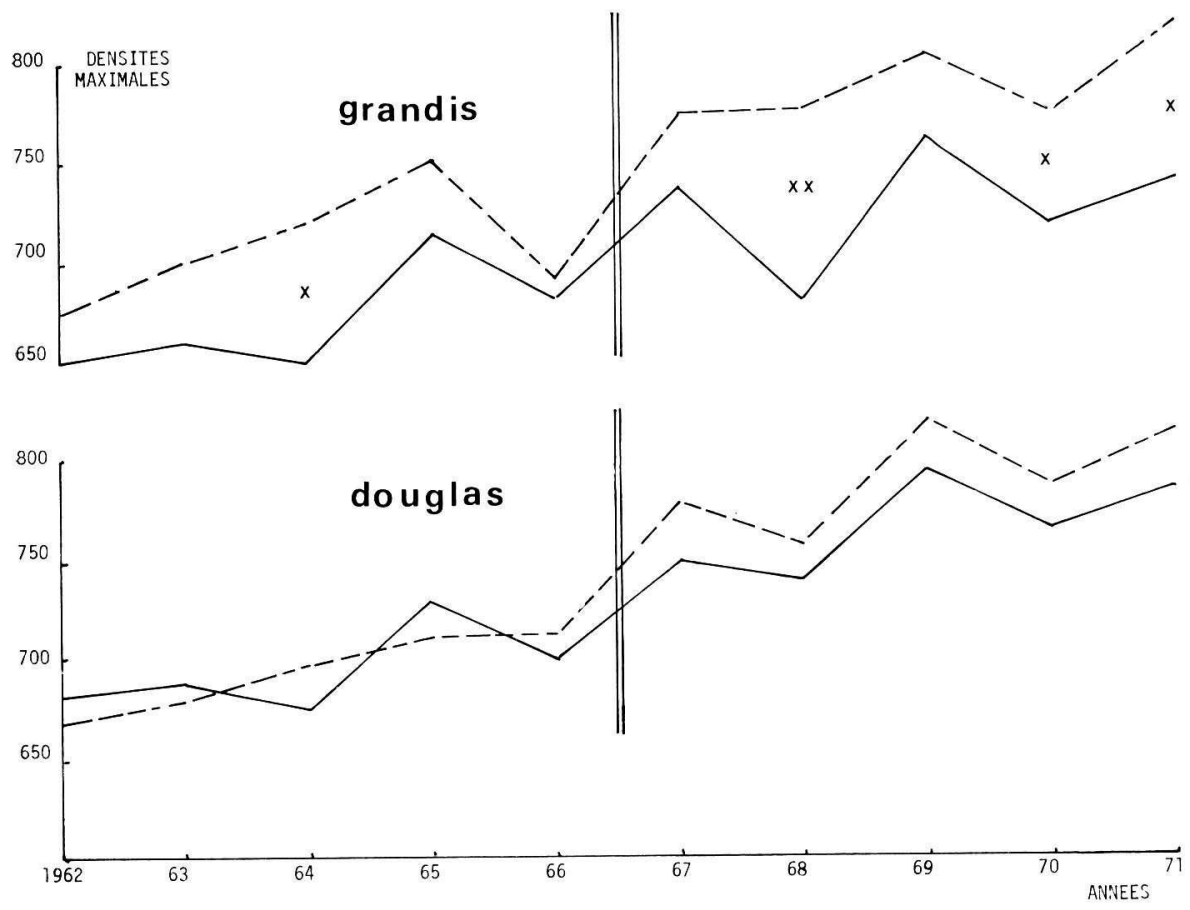

Fig. 5
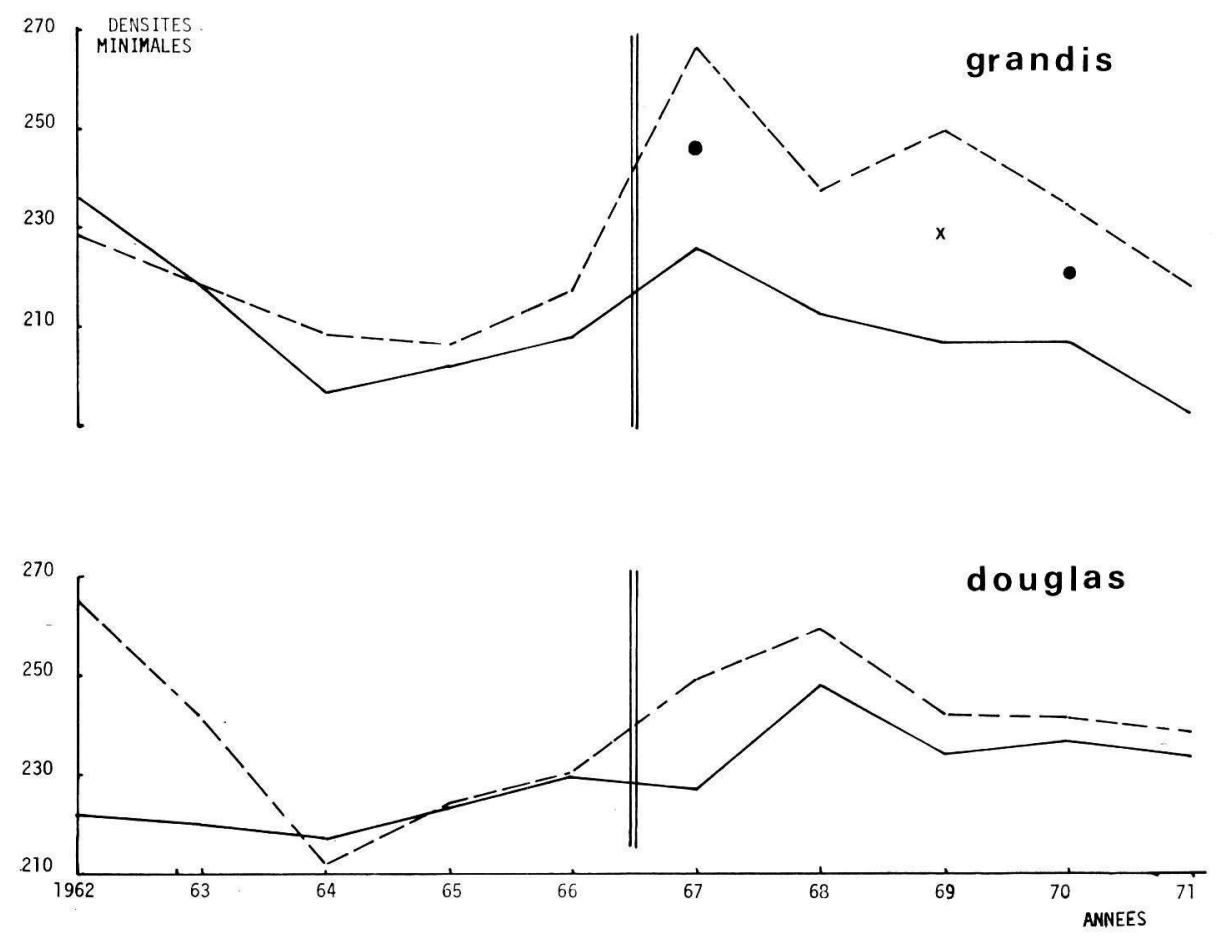

Fig .6 
Pour le douglas, aucune différence significative n'apparaît, mais, alors que l'écart était tantôt dans un sens, tantôt dans l'autre avant traitement, les valeurs des densités maximales des arbres élagués sont toujours supérieures à celles des témoins après traitement.

Accessoirement, la figure permet de constater la similitude des variations annuelles de la densité maximale entre les deux espèces (particulièrement pour les témoins), et également le gradient d'augmentation de la densité avec l'âge, très net chez le douglas.

\subsection{Densité minimale annuelle (figure 6)}

Ni chez le grandis, ni chez le douglas n'existait de différence significative avant l'année de l'élagage; par la suite, on observe pour l'un et pour l'autre une action positive du traitement, non significative chez le douglas et légèrement significative chez le grandis.

Contrairement aux densités maximales, l'effet des variations annuelles du climat n'est pas le même pour les deux espèces, et celui de l'âge n'est également marqué, ni pour l'une, ni pour l'autre.

\subsection{Indice de progressivité (figure 7)}

Cet indice qui, rappelons-le, traduit le caractère plus ou moins abrupt du passage du bois initial au bois final, avait avant traitement des valeurs très voisines pour les arbres destinés à être élagués et pour les futurs témoins; l'effet du traitement est spectaculaire, la transition devenant beaucoup plus progressive sur les individus ayant conservé la totalité de leur couronne vivante que sur ceux ayant subi une amputation sur la moitié de leur hauteur; l'influence de l'élagage est plus marquée initialement chez le grandis, mais paraît se poursuivre plus longtemps chez le Douglas.
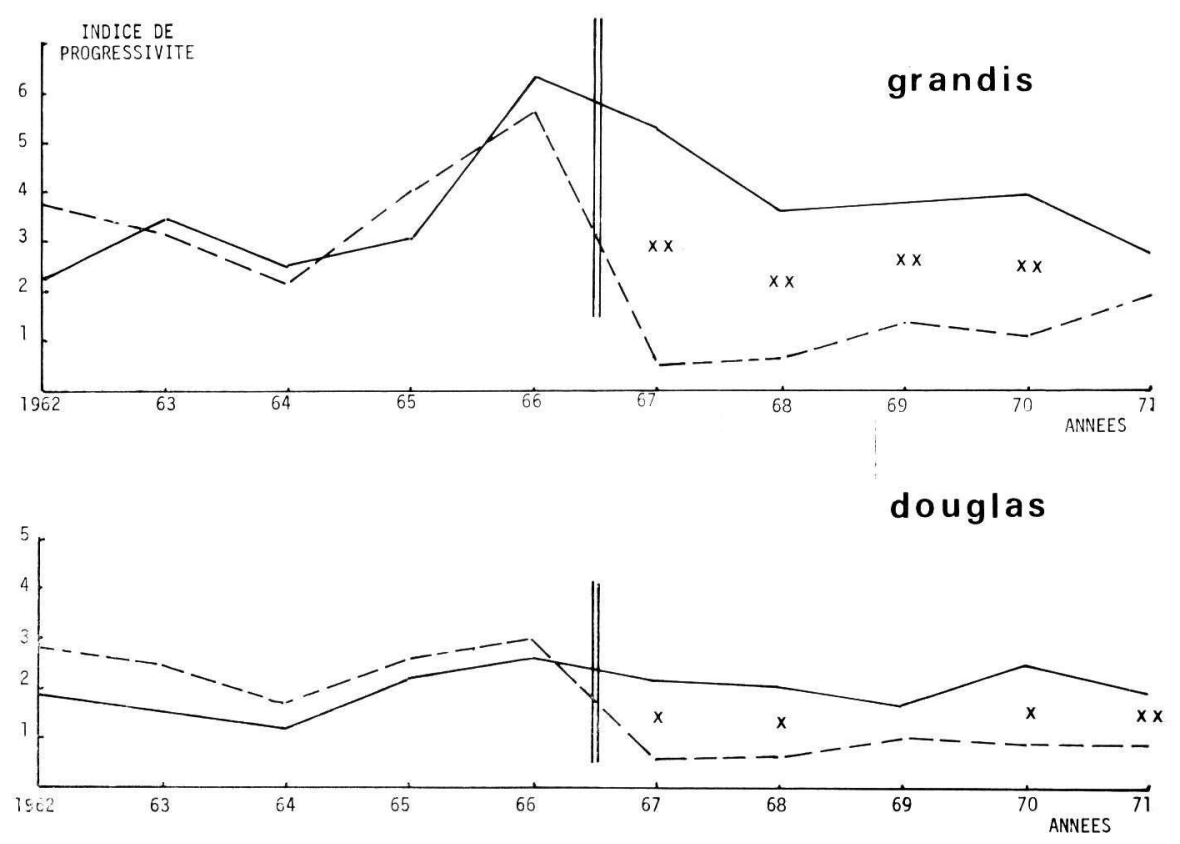

Fig, 7 


\subsection{Doubles cernes (figure 8)}

On n'observe que des tendances, mais qui vont dans le même sens pour les deux espèces : chez le grandis, à une homogénéité avant traitement correspond une hétérogénéité après traitement avec des doubles cernes plus nombreux et plus importants sur les arbres élagués que sur les témoins; chez le douglas, la supériorité de la note des doubles cernes observée sur les témoins avant traitement disparaît après celui-ci.

\section{NOTE FREQUENCE}
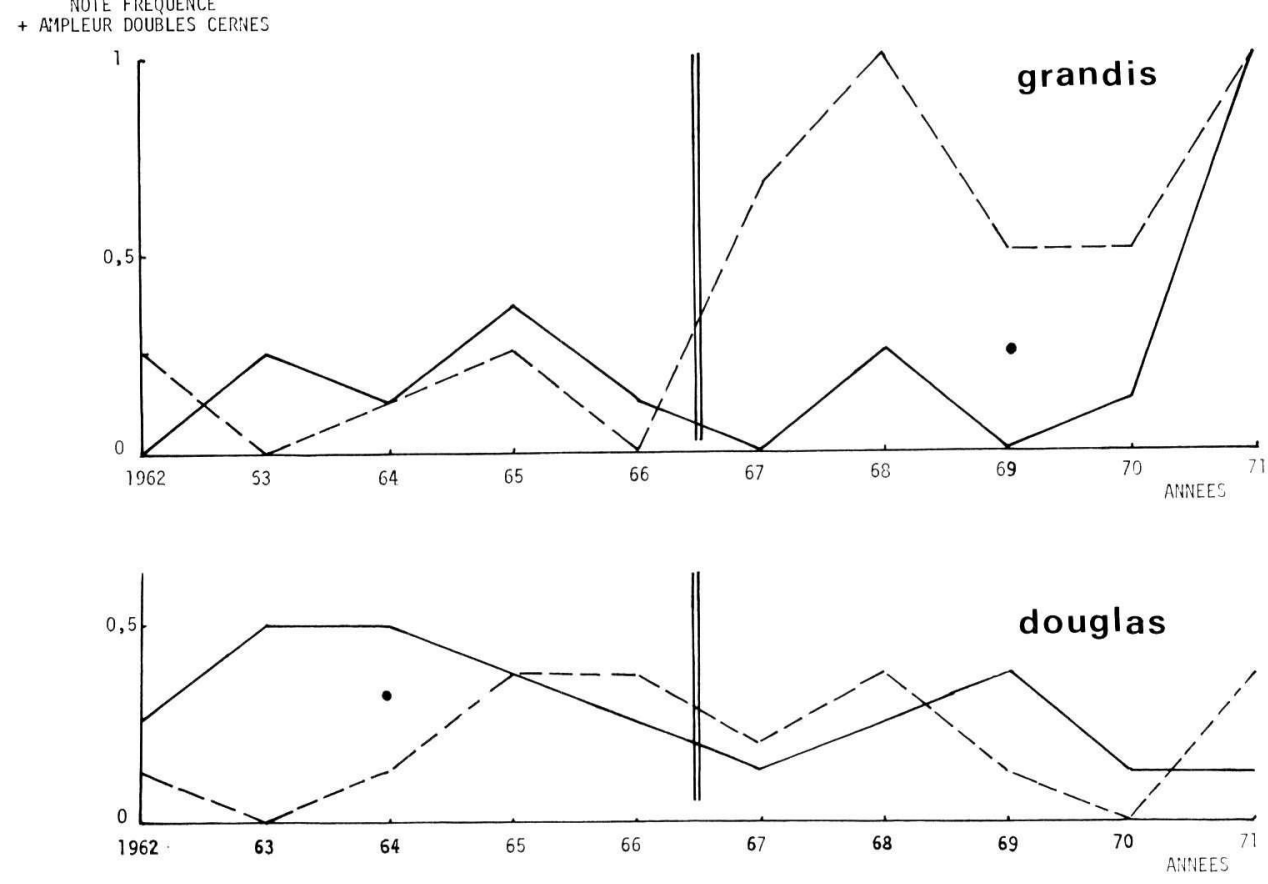

Fig. 8

\section{2. - Retraits}

\subsection{Observations relatives aux dimensions à l'état saturé}

Les résultats sont regroupés dans le tableau ci-contre où sont indiquées, dans chaque cas, les valeurs moyennes en microns :

Mais si l'on fait abstraction des tensions de croissance dont on sait qu'elles sont importantes mais très difficiles à mesurer, on peut admettre que les changements de dimensions à l'état saturé sont liés à la libération des contraintes exercées par la masse de l'arbre sur le volume théoriquement cylindrique de la carotte, calibré au diamètre de la tarière, et, qu'en conséquence, les dimensions saturées sont d'autant plus élevées que la contrainte subie était plus élevée.

Les remarques que l'on peut faire sur les chiffres ci-contre montrent que cette hypothèse se vérifie dans la plupart des cas; ainsi : 
TABLEAU 1

\begin{tabular}{|c|c|c|c|c|c|}
\hline & & \multicolumn{2}{|c|}{ Témoins } & \multicolumn{2}{|c|}{ Élagués à $50 \%$} \\
\hline & & $1962-1966$ & $1967-1971$ & $1962-1966$ & $1967-1971$ \\
\hline \multirow{4}{*}{$\begin{array}{l}\text { DOUGLAS } \\
\text { GRANDIS }\end{array}$} & Diamètre axial $\ldots \ldots \ldots$ & 5048 & 5040 & 5014 & 5004 \\
\hline & Diamètre tangentiel ..... & 4972 & 4955 & 4958 & 4914 \\
\hline & Diamètre axial $\ldots \ldots \ldots$ & 5044 & 5034 & 4983 & 4975 \\
\hline & Diamètre tangentiel $\ldots \ldots$ & 4938 & 4946 & 4885 & 4914 \\
\hline & $\begin{array}{l}\text { Diamètre axial } \\
\text { Diamètre tangentiel } \\
\text { Témoins } \\
\text { Élagués à } 50 \%\end{array}$ & $\begin{array}{l}=\text { Axial di } \\
=\text { Tangent } \\
=\text { Control } \\
=\text { Pruned }\end{array}$ & $\begin{array}{l}\text { tiameter } \\
0 \% \text { of the to }\end{array}$ & height & \\
\hline
\end{tabular}

- la contrainte axiale est toujours supérieure à la contrainte tangentielle,

- pour une même caractéristique et un même traitement, elle est toujours plus forte chez le douglas à bois dense que chez le grandis à bois léger,

- pour une même tranche de cernes, la contrainte est plus élevée sur les témoins que sur les arbres élagués, et ceci dans les deux directions et pour les deux espèces. L'enlèvement d'une masse importante de branches se traduit ainsi par une diminution de la charge exercée, aussi bien sur le bois formé après l'opération que sur celui des années précédentes; il n'y a là rien de surprenant car, du fait de la conicité de la tige, la composante verticale de la charge représentée par le houppier ne se confond pas avec le fil du bois,

- la contrainte axiale diminue toujours de la moelle vers l'écorce, ce qui peut également être considéré comme un effet de la conicité; il en est de même pour la contrainte tangentielle chez le douglas, mais pas chez le grandis.

\subsection{Observations relatives aux retraits proprement dits}

Sur le tableau ci-dessous sont données en \% les valeurs moyennes des retraits dans les

TABLEAU 2

\begin{tabular}{|c|c|c|c|c|c|c|}
\hline & \multicolumn{3}{|c|}{ Témoins } & \multicolumn{3}{|c|}{ Élagués à $50 \%$} \\
\hline & $1962-1966$ & $1967-1971$ & Différence & $1962-1966$ & $1967-1971$ & Différence \\
\hline DOUGLAS $\left\{\begin{array}{l}\text { Retrait axial ..... } \\
\text { Retrait tangentiel } \\
\text { Retrait radial.... }\end{array}\right.$ & $\begin{array}{l}0,352 \\
5,192 \\
2,812\end{array}$ & $\begin{array}{l}0,259 \\
6,201 \\
3,100\end{array}$ & $\begin{array}{r}-0,094 \\
+\quad 1,009 \\
+\quad 0,228\end{array}$ & $\begin{array}{l}0,174 \\
4,877 \\
2,562\end{array}$ & $\begin{array}{l}0,012 \\
6,337 \\
3,487\end{array}$ & $\begin{array}{l}-0,161 \\
+1,460 \\
+0,925\end{array}$ \\
\hline GRANDIS $\left\{\begin{array}{l}\text { Retrait axial .... } \\
\text { Retrait tangentiel } \\
\text { Retrait radial.... }\end{array}\right.$ & $\begin{array}{l}0,194 \\
5,146 \\
1,450\end{array}$ & $\begin{array}{l}0,304 \\
5,969 \\
1,187\end{array}$ & $\begin{array}{r}+0,110 \\
+0,822 \\
-0,263\end{array}$ & $\begin{array}{r}0,151 \\
4,479 \\
1,362\end{array}$ & $\begin{array}{r}0,175 \\
7,116 \\
2,175\end{array}$ & $\begin{array}{l}-0,024 \\
+2,637 \\
+0,813\end{array}$ \\
\hline
\end{tabular}


trois directions pour les 5 années précédant celle de l'élagage et pour les 5 années suivantes, ainsi que les valeurs algébriques des différences entre la seconde et la première période.

\subsection{Retrait axial}

Pour le douglas, le retrait axial diminue de la moelle vers l'écorce, aussi bien sur les témoins que sur les arbres élagués à $50 \%$; il y a donc un effet de l'âge (passage au bois adulte) indépendant de l'élagage; cependant, l'amélioration est plus nette pour les arbres élagués que pour les témoins, mais la différence entre la réduction du retrait axial des uns et des autres n'est pas significative $(t=0,59$ pour 14 degrés de liberté).

Pour le grandis, il y a aggravation du retrait axial sur les témoins et de la dilatation axiale sur les arbres élagués à $50 \%$; bien que l'évolution de la rétractibilité se fasse en sens contraire, l'effet de l'élagage n'est pas significatif $(\mathrm{t}=0,66)$.

\subsection{Retrait tangentiel}

Le retrait tangentiel pour la période 1967-1971 est toujours supérieur à celui de la période précédente; il y a là une influence du vieillissement de l'assise génératrice qui est indépendante de l'élagage.

Cependant, l'aggravation de la rétractibilité tangentielle, c'est-à-dire la différence de retrait entre la deuxième tranche de cernes et la première, est pour les deux espèces plus forte sur les arbres élagués que sur les témoins; la différence n'est pas significative pour le douglas $(t=1,01)$, mais elle est très significative pour le grandis $(t=4,37)$, et pour cette essence, il y a donc un effet «élagage » qui s'ajoute à l'effet «âge » et qui se traduit par une accélération du passage du bois juvénile au bois adulte.

\subsection{Retrait radial}

On remarque entre les années 1962-1966 et 1967-1971 une augmentation du retrait radial pour tous les douglas, ainsi que pour les grandis élagués, mais une diminution chez les grandis témoins; l'effet de l'âge sur cette caractéristique est donc variable, mais si l'on compare les différences entre les deux tranches de cernes successives des arbres élagués à celles des témoins, on observe toujours une aggravation qui est très significative, à la fois pour les douglas $(t=3,68)$ et pour les grandis $(\mathrm{t}=10,75)$; l'effet propre de l'élagage est donc ici très marqué.

\subsection{Observations relatives à l'infradensité}

Les valeurs moyennes de cette caractéristique sont reportées sur le tableau ci-dessous :

TABLEAU 3

\begin{tabular}{|c|c|c|c|c|}
\hline \multirow{2}{*}{ Infra-densité } & \multicolumn{2}{|c|}{ Témoins } & \multicolumn{2}{|c|}{ Élagués à $50 \%$} \\
\hline & $1962-1966$ & $1967-1971$ & $1962-1966$ & $1967-1971$ \\
\hline $\begin{array}{l}\text { Douglas } \ldots \ldots \ldots \ldots \ldots \\
\text { Grandis } \ldots \ldots \ldots \ldots \ldots\end{array}$ & $\begin{array}{l}369,7 \\
291,4\end{array}$ & $\begin{array}{l}370,6 \\
282,2\end{array}$ & $\begin{array}{l}373,2 \\
283,8\end{array}$ & $\begin{array}{l}424,7 \\
334,9\end{array}$ \\
\hline
\end{tabular}


Il n'existe de différence significative, ni entre les deux tranches de cernes des témoins, ni entre l'une ou l'autre d'entre elles et la tranche 1962-1966 des arbres élagués; en revanche, l'augmentation de la densité sur les arbres dont $50 \%$ de la hauteur du houppier ont été enlevés est très significative, aussi bien pour le douglas $(t=4,06)$ que pour le grandis $(\mathrm{t}=5,03)$; on a ainsi pour la densité globale une influence très marquée de l'élagage n'interférant en rien avec celle de l'âge puisque l'effet de celui-ci est absolument négligeable.

\section{4. - COMMENTAIRES}

Quelques anomalies dans les résultats demeurent difficiles à expliquer; elles concernent d'ailleurs toutes le grandis; ainsi, ne trouve-t-on pas de raisons valables pour rendre compte de l'augmentation avec l'âge de la contrainte tangentielle pour cette essence, ou encore de l'aggravation des changements de dimensions dans le sens axial de la moelle vers l'écorce.

En revanche, la mise en évidence sur les grandis élagués à $50 \%$ de retraits négatifs (donc d'expansion du bois lorsque le taux d'humidité diminue) ne doit pas surprendre outre mesure car le phénomène a déjà été mentionné pour d'autres espèces (Anonyme, 1960 - HaNN, 1969); Barber et Meylan (1964) ont même prouvé mathématiquement, en partant d'un modèle théorique, que de tels retraits négatifs pouvaient se produire lorsque, à un angle important d'insertion des microfibrilles sur l'axe de fibres, s'ajoute un rapport élevé entre les modules d'élasticité de la partie cristalline et de la partie amorphe de la couche moyenne de la membrane secondaire; lorsque la zone non cristalline a une forte teneur en eau, son module d'élasticité est faible et, par suite, le rapport précité croît, entraînant la possibilité de retraits négatifs; l'observation faite sur les arbres élagués concorde assez bien avec cette théorie, puisqu'on peut admettre que l'enlèvement d'une partie importante de la couronne vivante a pour effet de réduire l'évapo-transpiration, donc d'augmenter la teneur en eau du bois.

Les autres résultats dont il est rendu comote ci-dessus sont dans leur ensemble très cohérents et tendent à montrer qu'un élagage sévère a pour effet d'accélérer le passage du bois juvénile au bois adulte, ce qui se traduit par un accroissement, aussi bien de l'infradensité que des densités maximales et minimales annuelles, par une réduction de la largeur des cernes et par une augmentation des retraits tangentiels et radiaux.

On peut observer également que, pour toutes les caractéristiques étudiées, l'influence du traitement est beaucoup plus marquée sur le grandis que sur le douglas; ceci est très satisfaisant car on sait que, d'une façon générale, les caractéristiques technologiques du premier sont bien inférieures à celles du second; de ce point de vue, l'amélioration apportée par l'élagage est particulièrement bienvenue, et l'on peut même dire que l'enlèvement d'une partie relativement importante du houppier permet d'obtenir avec l'Abies grandis un bois qui se rapproche qualitativement de celui du Sapin pectiné.

Certes, la baisse de l'indice de progressivité occasionnée par l'élagage est en elle-même regrettable, mais cette opération ne fait vraisemblablement qu'en avancer la date sans en modifier l'ampleur, et le phénomène se produirait de la même façon lors du passage normal du bois juvénile au bois adulte. Il en est de même pour les retraits tangentiels et radiaux qui au surplus, n'atteignent jamais une valeur excessive; leur aggravation est d'ailleurs en partie 
compensée par la diminution de la rétractibilité axiale qui est faible en valeur relative, mais très avantageuse dans la pratique car elle intéresse la direction du fil du bois qui est celle de la plus grande longueur des débits.

Reçu pour publication en mars 1973.

\section{REMERCIEMENTS}

L'appareil de mesure du retrait a été initialement construit par R. COCHARD, alors Technicien à la Station, et grandement amélioré par J. R. PERRIN qui lui a succédé après son départ à la retraite.

Le traitement des données a été fait à la Station de Biométrie du C. N. R. F. suivant un programme spécifique conçu par C. MILLIER.

Que tous trouvent ici l'expression de nos meilleurs remerciements pour l'aide précieuse qu'ils nous ont ainsi apportée.

\section{SUMMARY}

EFFECT OF GREEN PRUNING ON RING STRUCTURE AND ON CERTAIN WOOD FEATURES

OF DOUGLAS FIR AND GRAND FIR

The influence of heavy green pruning on wood quality is investigated from 16 trees pruned at $50 \%$ of the total height and 16 control trees ( 8 Douglas fir and 8 Grand fir for each treatment).

The material was $5 \mathrm{~mm}$ increment cores taken at breast height.

On X-ray photographs densitometric records were measured the following data :

- Ringwidth,

- Maximum annual densities,

- Minimum densities, fig. 1),

- Progressivity index at the transition between earlywood and latewood (see parag. 22 and

- False ring index (see fig. 2 and 2 bis).

Elsewhere shrinkage values were computed from about 100 measurements per tree and per direction made at an accuracy of $1 \mu$ with an electronic gauge (fig. 3 ) connected to a teletype which gives a listing of the data and transfer them on a punched tape.

Due to pruning the ringwidth and the progressivity index decrease (fig. 4 and 7), the maximum and minimum annual densities (fig. 5 and 6) and the false ring index increase (fig. 8).

The measurements of core sizes in wet conditions give useful indications about the internal stresses of the tree with respect to age and to treatment (table 1).

Pruning increases significantly both tangential and radial shrinkage and decreases the longitudinal shrinkage of Douglas fir; the effect on longitudinal shrinkage of Grand fir is not very clear (table 2).

These results show that pruning causes an acceleration of the change from juvenile wood to mature wood.

\section{ZUSAMMENFASSUNG}

DER EINFLUSS DER GRÜNASTUNG AUF DIE STRUKTUR DER

JAHRINGE UND AUF EINIGE ANDERE HOLZMERKMALE BEI DER KÜSTENTANNE UND DER DOUGLASIE

Der Einfluss einer starken Grünastung (Entnahme von $50 \%$ der Krone) auf verschiedene Holzmerkmale der Douglasie und der Küstentanne wurde an $5 \mathrm{~mm}$ dicken Bohrspänen aus 1,30 m Höhe untersucht. Von jeder der beiden Holzarten standen jeweils acht aufgeastete und acht unbehandelte Bäume zur Verfügung. men :

Den densitometrischen Registrierungen der Röntgenaufnahmen wurden folgende Daten entnom- 
- Jahrringbreite,

- Dichtemaximum des Jahres,

- Dichteminimum des Jahres,

— Index der Übergangs vom Früh- zum Spätholz (siehe Abschn. 22, Abb. 1),

- Index der Doppelringe (siehe Abb. 2 und Abb. 2 bis).

Desweiteren wurden die Werte der Schwindung in verschiedenen Richtungen mit einem elektronischen Weggeber (Präzision : $1 \mu$ ) gemessen und automatisch auf einen Fernschreiber übertragen, welcher die Daten in Klarschrift und auf Lochstreifen wiedergibt.

Pro Baum und Richtung wurden jeweils 100 Messungen durchgeführt.

Die Grünastung führte zu einer Abnahme der Jahrringbreiten (Abb. 4) und des Übergangindexes (Abb. 7), verursachte eine Zunahme der jährlichen Dichtemaxima und Minima (Abb. 5 und 6) und des Doppelringindexes (Abb. 8).

Die Messungen an wassergesättigten Bohrspänen geben interessante Aufschlüsse über die bauminternen Spannungen in Abhängigkeit von Alter und Behandlung (Tab. 1).

Die Grünastung erhöht signifikant die radiale und tangentiale Schwindung und vermindert die Axiale Schwindung bei der Douglasie. Die Abnahme der axialen Schwindung ist bei der Küstentanne nicht sehr deutlich ausgeprägt (Tab. 2).

Die vorliegenden Ergebnisse zeigen, dass die Grünastung eine Beschleunigung des Überganges vom Früh- zum Spätholz hervorruft.

\section{RÉFÉRENCES BIBLIOGRAPHIQUES}

Anonyme, 1960. - Longitudinal shrinkage of Wood. Forest Products Laboratory, Madison, report $n^{0} 1093$.

Barber N. F. et Meylan B. A., 1964. - The anisotropic shrinkage of wood. A theoretic model. Holzforschung, 18 (5), 146-156.

Brown G. S., 1965. - The yield of clearwood from pruning : some results with Radiata Pine. Commonwealth Forestry Review, vol. 44 (3), no 121.

Callin G., 1966. - Economics of stem pruning. Department of Operational Efficiency, Royal Swedish College of Forestry, Stockholm, Research Note no 28 , p. 71-74, Chapter 5.

Feinl O. et Godin V., 1965. - Plantation-grown red pine for veneer and plywood, Canadian Wood Products Industries, janvier.

Fielding J. M., 1964. - The pruning of plantation-grown pines. Commonwealth Forestry Review, vol. $43(4), \mathrm{n}^{\circ} 118$.

Gava M., 1969. - Cercetari privind aplicarea elagajului artificial la molid si brad. Institutul de cercetari forestiere, Bucarest.

Gerischer G. F. R. et de Villiers A. M., 1963. - The effect of heavy pruning on timber properties. Forestry in South Africa, no 3, 15-41.

HANN R. A., 1969. - Longitudinal shrinkage in seven species of wood. Forest Products Laboratory, Madison, U. S. D. A. Forest Service, Research Note FPL-0203.

Kano T., Sudo S. et NaKagawa S., 1969. - On the wood quality of plantation-grown Akamatsu (Pinus densiflora). Bulletin of the Government Forest Experiment Station no 219, Tokyo.

Keller R., 1968. - L'élagage artificiel de branches vivantes sur résineux. Revue Forestière Française, n' $7-8$, juillet-août.

LARSON P. R., 1961-1962. - A biological approach to wood quality. Tappi vol. 45, no 6 et vol. 46 , $\mathrm{n}^{\circ} 6$.

LARSON P. R., 1966. - Stem form of young larix as influenced by wind and pruning. For. Sci., 11 (4), 412-24.

LehtPERE R., 1957. - Influence of high pruning on the growth of Douglas fir. Forestry, 30, 1.

NAGELI V., 1952. - Pruning experiments in even-aged conifer stands of central Zwitzerland. Mitt. Schweiz. Anstalt für Forts. Versuchs, 28, 271-354.

Polge H., 1967. - Premiers résultats de l'expérience d'étalage de branches vivantes d'Épinal. Revue Forestière Française, $\mathrm{n}^{0} 8-9$, août-septembre.

Polge H., 1969. - Densité de plantation et élagage de branches vivantes, ou Pourquoi, Quand et Comment élaguer? Revue Forestière Française, XXI, n ${ }^{\circ}$ spécial « Sylviculture ».

VENET J., 1955. — L’élagage artificiel des peuplements résineux. Revue Forestière Française, no 8, 607-620.

Venet J., 1957. — L'élagage artificiel des peupliers et la production de bois de choix. Actes du VIe Congrès International du Peuplier, Paris, 413-423. 